\title{
Quality Improvement Project: Implementation of PACS Integrated Peer Feedback for Radiologists After an Hospital Merge
}

\author{
Allard Olthof ${ }^{1, *}$ \\ ${ }^{1}$ Treant healthcare group, Netherland \\ "Corresponding author: Allard Olthof. E-mail: a.olthof@treant.nl
}

Received 2016 December 21; Accepted 2017 February 08.

\begin{abstract}
Objectives: Peer feedback among radiologists contributes to quality improvement. RadPeer is a commonly used system for peer review. Consensus-oriented group review (COGR) promotes discussion among radiologist to improve reporting quality of all team members. Thresholds to feedback can be technical and personal, that can be augmented by a hospital merge. Making use of the functionality of the picture archiving and communication system (PACS) can help to integrate peer feedback in daily workflow. A good working agreement can augment usage of the feedback system by radiologists. The purpose of this paper is to demonstrate the implementation of PACS integrated peer feedback after an hospital merge with 3 locations.

Methods: The workflow for peer feedback was designed and the project was approved by all radiologists. We adapted the teaching file function of the PACS to use the RadPeer feedback terminology. The radiologists were instructed how to use the system. Team meetings were scheduled to discuss cases, in line with COGR.

Results: Providing and receiving peer feedback was easy to do, without interruption in daily workflow. The system can be used among the three hospital locations of the healthcare group. It was easy to retrieve and demonstrate cases for group discussion and analysis. A side effect was that the feedback project helped to demonstrate to external stakeholder that the radiologists are eager to work on continuous quality improvement.

Conclusions: PACS integrated peer feedback is a valuable tool to improve quality in radiology and feasible to implement, especially in the setting of a hospital merge.
\end{abstract}

This is an abstract presented in the 33rd Iranian congress of radiology (ICR) and the 15th congress of Iranian radiographic science association (IRSA). 